\title{
Performance Evaluation of Different Wavelet Families for Chromosome Image De-Noising and Enhancement
}

\author{
SaumyaDubey, Deepak Tiwari, O.P. Singh, Ashish Dixit \\ Department of Electronics \& Communication Engineering Amity School of Engineering \& \\ Technology Amity University Uttar Pradesh, Lucknow Campus, India
}

\begin{abstract}
Bio-medical image de-noising and image enhancement are the two necessary and noticeable eras for better diagnosis and therefore the treatment within the medical field. Chromosomes images are ever found as blur images, so de-noising and image enhancement becomes essential extremely and area of interest for the researchers. Here the work of this analysis paper accommodates the chromosome image de-noising with the assistance of 'Discrete Wavelet Transform (DWT)' families one by one and additionally the performances of different wavelet families are evaluated based on their Peak Signal to Noise Ratio (PSNR) and the value of Mean Square Error (MSE). After this, the three enormously efficient image improvement techniques are applied e.g. Contrast Adjustment (CA), Histogram Equalization (HE) \& Adaptive Histogram Equalization (AHE) for the betterment of visualization. This single chromosome image could be a 2-D image. With the use of widely applicable 'coif5'Discrete Wavelet Transform, the chromosome image is decomposed up to the level 2 , threshold and de-noised.

Image enhancement is an important development to boost the visual impact of any image. All the simulation is performed with the help of the MATLAB R2010a platform.
\end{abstract}

Keywords: DWT, De-noising, Thresholding, Contrast Adjustment, Histogram Equalization, Adaptive Histogram Equalization, MATLABR2010a.

\section{INTRODUCTION}

To extract the correct information from the medical signals is always the area of interest for the researchers. There are so many bio-medical signals to de-noise for the better treatment of patients in medical science i.e. ECG and ICG signals etc [1], [2].

Chromosome imaging has its vital role in chromosome classification and in the technique which describes the chromosome detail indexes. Many unwanted sources are here which createnoise to any chromosome image. Chromosome image de-noising and enhancement will helpful for the medical practitioners or researchers. Conditions while accessing the picture, Staining and sample defects are the main factors due to which the noise comes along with the image. So here de-noising and visual enhancement of chromosome image are necessary for its further applications. Wavelet is an important tool for signal processing [1]. Wavelet is applied due to its multiresolution properties.

For the process of de-noising, in this paper coif5 is employed and decomposition is performed on second level. This is also a matter of concern to select the family type of wavelet and decomposition level [2]. Here one more phenomenon known as thresholdingselection criterion which also plays a major part for evaluating the performance of de-noising method [3].

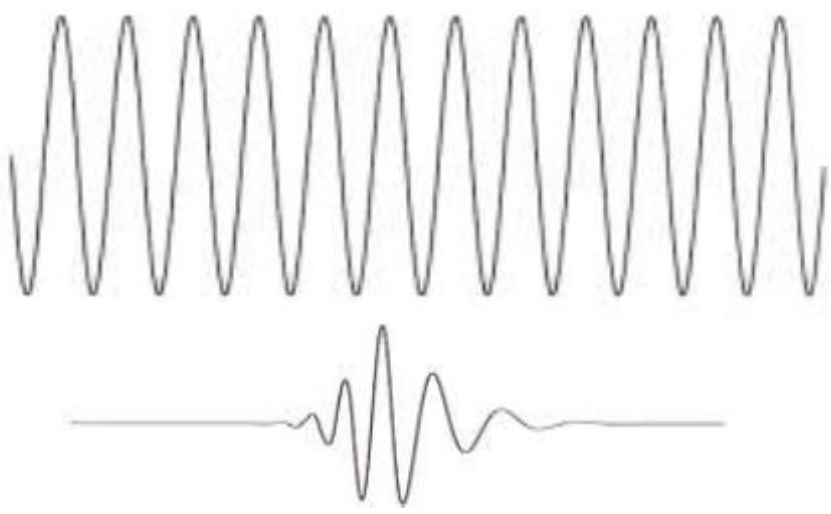

Figure 1: Demonstration of (a) Wave and (b) Wavelet 


\section{Discrete Wavelet Transform (DWT):}

Transformation is the tool to convert any signal from one domain to another because sometimes it is convenient to analyze the signal different from present domain. Redundancy comes out from the DWT is less with compare to CWT. This gives a discrete analysis to the signal by scaling and translating it in a discrete manner.

$$
\operatorname{DWT}\left(\tau, \mathrm{s}_{0}\right)=\frac{1}{\sqrt{\mathrm{s}_{0}^{\mathrm{j}}}} \int_{-\infty}^{\infty} \mathrm{f}(\mathrm{t}) \Psi\left(\frac{t-k \tau_{0} s_{0}^{j}}{s_{0}^{j}}\right) d t
$$

Where scaling and translating factors $\mathrm{k}, \mathrm{j}$ are just integers. The mother wavelet is represented in the term of scaling and translational of dyadic transform as[4];

$$
\Psi_{j, k}(t)=2^{-\frac{j}{2}} \varphi\left(2^{-j}\right) t-k
$$

After de-noising the image it is also an important constraint to enhance the image for the better visual effect.

\section{Imageenhancement:}

Enhancement is also a matter of concern for the chromosome images. The image adjustment technique adjust the contrast of the image. In HE the histogram of the image is equalized and in AHE it equalize the histogram but follow the pattern of original histogram.In section II the de-noising method with the help of DWT is defined, image enhancement is discussed in the section III, section IV, V respectively shows the result and discussion and conclusion of this paper.

\section{II. .CHROMOSOME IMAGE DENOISING}

Noise reduction method is an important feature of image processing. This procedure recovers the original signal that has been corrupted by undesirable signals or noise. Here for understanding the results clearly, noise is added to the image. De-noising algorithm is used here with different-different wavelet families, some important steps are followed for accomplishing the de-noising process.

\section{De-noising Algorithm:}

1). First of all a random noise is added in the original image.

2). Apply DWT decomposition up to the level two with db4.

3). Now apply soft thresholding with four different thresholding selection criteria namely heursure, rigrsure, minimaxi and sqtwolog using global median estimator.

4). Here de-noised image is obtained then this whole algorithm is repeated by applying sym4, coif5 and haar wavelets in step two, replacing db4.

\section{MAGE DECOMPOSITION:}

Discrete wavelet transform decomposes the original data into different scaleby the use of wavelet filter banks[5]. The DWT uses two types of filters in this method. First is low pass filter, which is denoted by $\mathrm{G}_{\mathrm{o}}$ and second is high pass filter denoted by $\mathrm{H}_{0}$, after the whole process we get some coefficients. The approximation coefficients are obtained from low pass filter and detailed coefficients from high pass filter. These are the output coefficients and down sampled by 2 at each level. These coefficients are further used for the next process as an input details.

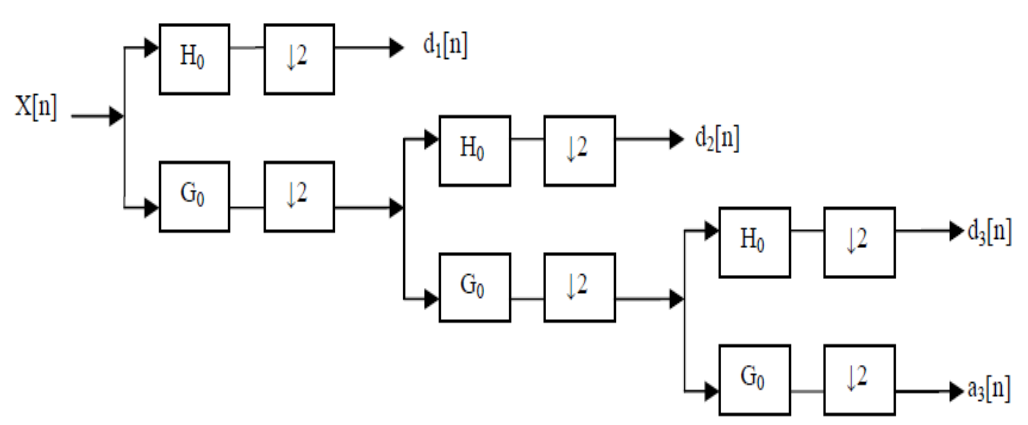

Figure 2: Three Level Decomposition Tree

By the selection of mother wavelet in DWT , the noisy image is decomposed, taking the decomposition level up to 2. We obtained the approximation coefficients a[n] and the detailed coefficients $d[n]$, which are shown in above figure. 


\section{THRESHOLDING:}

After the decomposition process, thresholding of obtained output coefficients are done to remove the coefficients below the certain value called as threshold value. This process removes the low amplitude noise or any noise overlap[6].

It is necessary to select the type of thresholding and this is decided by the requirement of application. Two types ofthresholding are HARD and SOFT, Which can be mathematically expressed as:-

\section{Hard threshold:}

Soft threshold:

$$
\left\{\begin{array}{cc}
\text { dj' }=\text { dj } & \text { if }|\mathrm{dj}|>T \\
\text { dj' }=0 & \text { if }|\mathrm{dj}|=<T
\end{array}\right.
$$

$$
\begin{cases}\mathrm{dj}^{\prime}=\operatorname{sign}(\mathrm{dj})(|\mathrm{dj}|-\mathrm{T}) & \text { if }|\mathrm{dj}|>T \\ \mathrm{dj}{ }^{\prime}=0 & \text { if }|\mathrm{dj}|=<T\end{cases}
$$

From the above described formula of soft thresholding, we are getting the estimated wavelet coefficients $d_{j}$. At each level a threshold value is found using the thresholding criteria and this value is applied for detailed coefficients $\mathrm{d}_{\mathrm{j}}$ by adopting Global median estimator[7],[8]. Soft thresholding does not produce discontinuities but in hard thresholding these discontinuities are appears often which are shown in figure 2. So the MATLAB platform by default takes the soft type thresholding for best results.
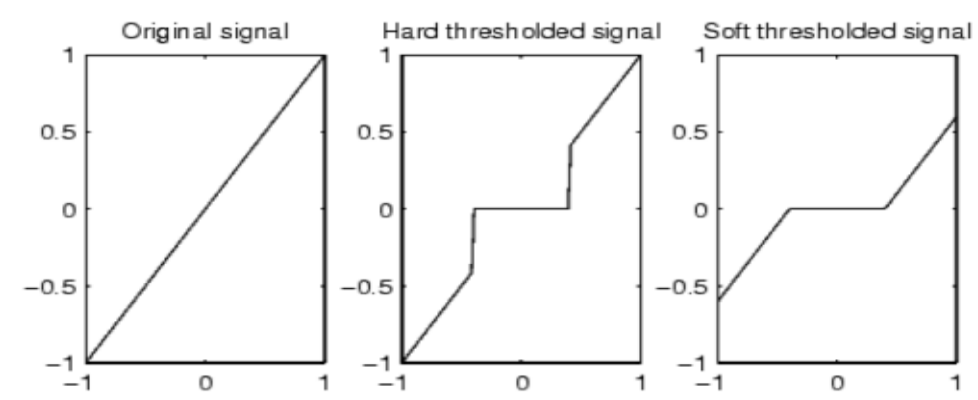

Figure 3: Threshold types: (a) Original signal; (b) Hard Thresholding; (c) Soft Thresholding

The Global threshold method is derived by D. Donoho's method. This universal threshold is given by below equation[9],[10]:- $\mathrm{T}=\sigma(2 \log \mathrm{L})^{112}$

Where, $\mathrm{T}$ denotes the threshold value, $\mathrm{L}$ is no. of samples or length of the signal and $\sigma$ is the standard deviation of noise. Now after de-noising the image, further the visual enhancement techniques are applied.

\section{CHROMOSOME IMAGE ENHANCEMENT}

Image Enhancement process consist of the collection of some techniques to improve the visual appearance of medical image or to convert the image for better analysis by a human or a machine [11]. Medical images suffer from poor contrast often, therefore it is important to enhance the contrast. Our aim is to enhance the chromosome image for increasing the image visibility and details. For this improvement Image Contrast Adjustment (CA), Histogram Equalization (HE) and Adaptive Histogram Equalization(AHE) methods[12]. These are described in the following steps,

\section{1). Image Adjustment:}

Image adjustment is the first step for adjusting the image[13]. This is shown by taking histogram of the adjusted image. Histogram provides a graphical representation of image contrast and brightness characteristics and is useful for evaluating contrast deficiencies like low or high [14]. It is a graphical plot, displays the input data values on the $\mathrm{x}$-axis and number of pixels in $\mathrm{y}$-axis. The histogram is a discrete function which is shown in below figure.

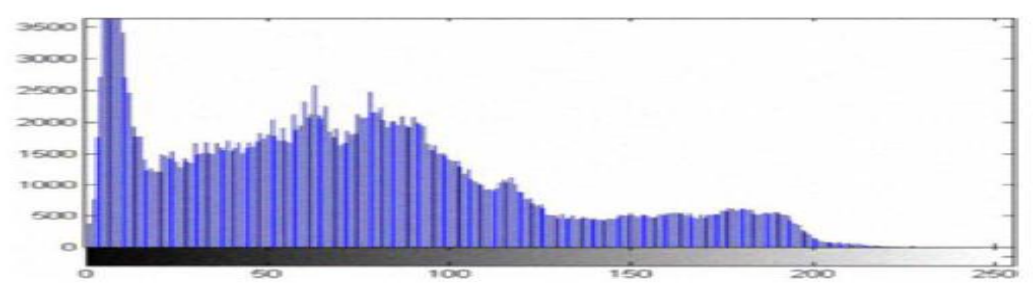

Figure 4: Histogram 


\section{2). Histogram Equalization:}

Histogram Equalization is the technique which changes the histogram of animage and redistributes all pixels uniformly, which is close to the desired histogram [15]. HE allows areas of lower contrast to obtain a higher contrast and automatically takes a transformation function to obtain an output image with a uniform Histogram. By using this adjustment, the intensities of image can be better distributed on the histogram [16].

\section{3). Adaptive Histogram Equalization:}

Adaptive Histogram equalization is different from simple histogram equalization. The adaptive method computes several histograms and redistributes them. It is suitable for improving the local contrast of an image and bringing out more detail [17].The improvement after this technique is shown in result section on chromosome image which is obtained by our simulation on MATLAB.

\section{RESULT AND DISCUSSION}

These results are showing the reliability of wavelet for de-noising the chromosome image and also the effectiveness of visual enhancement techniques. This section is going to be discussed in two part namely denoising results and enhancement results.

\section{1). De-noising Results:}

Here the results are showing that the procedure of work done is liable. In figure 5(a) the original image is shown of a single chromosome, this is a blur and noisy imagebut for clear observation of de-noising results we add some random noise in this image with noise variance 0.05 which is shown in figure 5 (b) then this noisy image is taken for de-noising by different-different wavelet families, according to the table1 and table 2 of PSNR and MSE results found from the coif5 family are better than other wavelet families so here in figure 5(c) the denoised image by coif5 is shown.

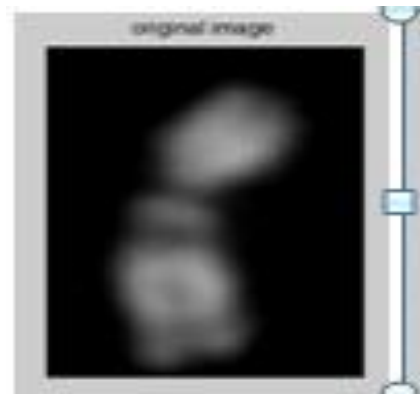

(a)

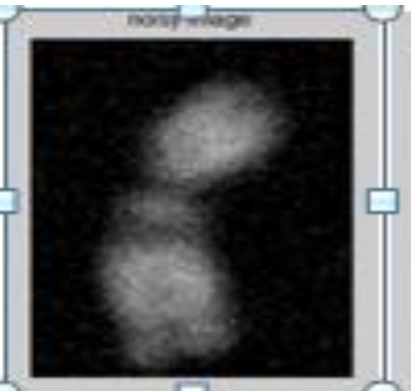

(b)

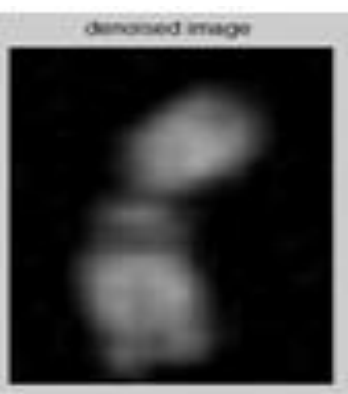

(c)

Figure 5: Chromosome De-noising Results

Table 1:PSNR results for different Wavelet Families at Different values of noise variance

\begin{tabular}{|l|l|l|l|l|}
\hline Noise Variance/Wavelet Families & $\mathbf{0 . 0 5}$ & $\mathbf{0 . 1 0}$ & $\mathbf{0 . 1 5}$ & $\mathbf{0 . 2 0}$ \\
\hline Db4 & 31.40 & 29.81 & 29.02 & 28.17 \\
\hline Sym4 & 31.44 & 30.00 & 29.12 & 26.05 \\
\hline Coif5 & 31.64 & 29.99 & 29.11 & 28.31 \\
\hline Haar & 29.47 & 27.71 & 28.39 & 27.78 \\
\hline
\end{tabular}

Table 2:MSE results for different Wavelet Families at Different values of noise variance

\begin{tabular}{|l|l|l|l|l|}
\hline Noise Variance/Wavelet Families & $\mathbf{0 . 0 5}$ & $\mathbf{0 . 1 0}$ & $\mathbf{0 . 1 5}$ & $\mathbf{0 . 2 0}$ \\
\hline Db4 & $1.92 \mathrm{e}-004$ & $7.9 \mathrm{e}-004$ & 0.0015 & 0.0025 \\
\hline Sym4 & $1.83 \mathrm{e}-004$ & $6.82 \mathrm{e}-004$ & 0.0014 & 0.0025 \\
\hline Coif5 & $1.50 \mathrm{e}-004$ & $6.91 \mathrm{e}-004$ & 0.0014 & 0.0025 \\
\hline haar & 0.001 & 0.0017 & 0.0020 & 0.0030 \\
\hline
\end{tabular}

Table 3:Performance of Global Median Estimator at Noise Variance 0.05

\begin{tabular}{|l|l|l|l|}
\hline Thresholding Criteria & PSNR(db) & MSE & Error \\
\hline Rigrsure & 31.26 & $2.222 \mathrm{e}-004$ & 0.0189 \\
\hline Heursure & 31.24 & $2.523 \mathrm{e}-004$ & 0.0365 \\
\hline sqtwolog & 31.18 & $2.831 \mathrm{e}-004$ & 0.0151 \\
\hline minimaxi & 31.27 & $2.183 \mathrm{e}-004$ & 0.0296 \\
\hline
\end{tabular}


Values found from table 3 also have its huge meaning because this table directs for the best thresholding selection criteria.

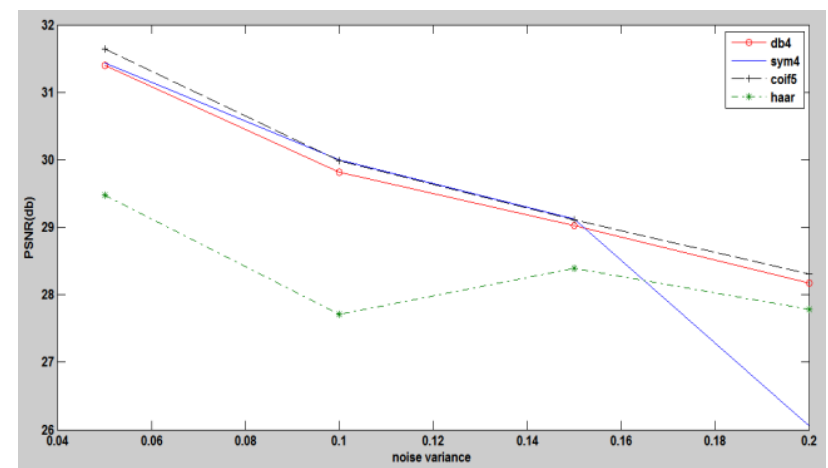

Plot 1:Comparative performance of different Wavelet Families between PSNR vs Noise Variance

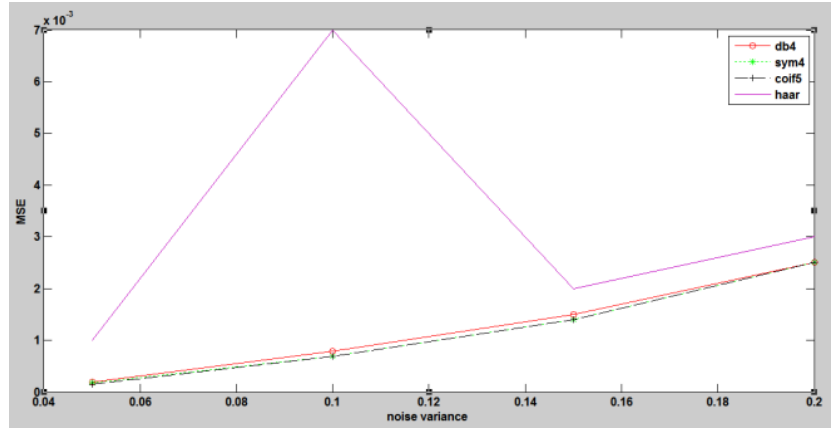

Plot 2:Comparative performance of different Wavelet Families between MSEvs Noise Variance

\section{2).Enhancement Results:-}

In this section the results of enhancement techniques applied on single chromosome image are shown here. Three techniques CA, HE and AHE are applied and shown in figure 6(a), 6(b) and 6(c) respectively along with their histogram representation.

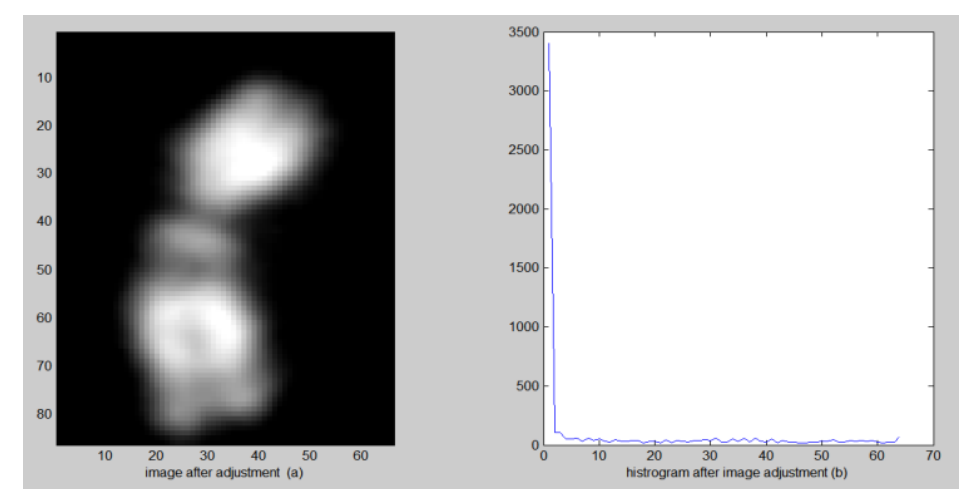

Figure 6(a): Chromosome Image after Contrast adjustment (CA)
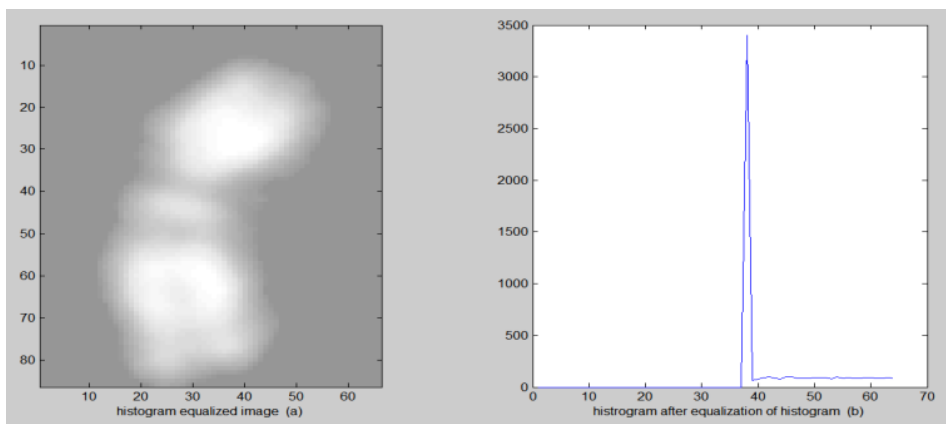

Figure 6(b): Chromosome Image after Histogram Equalization (HE) 

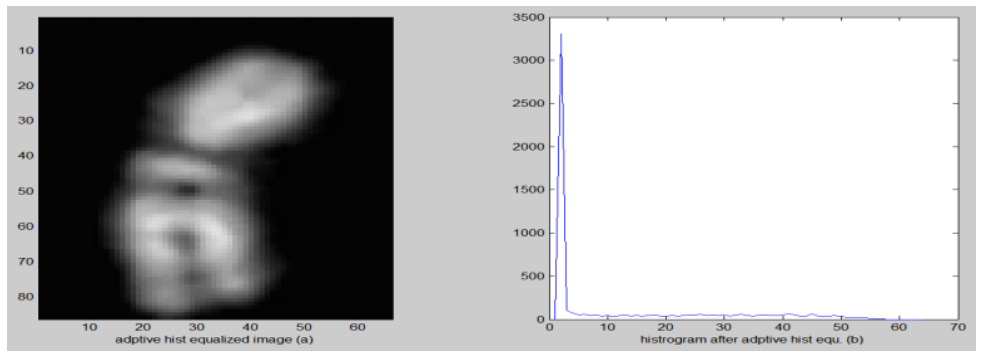

Figure 6(c): Chromosome Image after Adaptive Histogram Equalization(AHE)

Figure 6: Chromosome Enhancement results

\section{CONCLUSION}

On the basis of this research paper this can be concluded that the wavelet transform performs better than any other tool for de-noising the image. Here according to our method coif5 is giving the best results with rigrsurethresholding criteria and global median estimator. The three techniques discussed above also gives the better results for enhancing the visual effect of the image but among them Adaptive Histogram Equalization (AHE) gives the best result.

\section{REFERENCES}

[1]. R. C. Gonzalez and R. E. Woods, "Digital image processing", 2002,Prentice-Hall, USA.

[2]. O.P. Singh, K.K Singh, G.R. Mishra, Deepak Tiwari, SaumyaDubey, "Analysis of ECG Signaling Using Wavelet Transform", International Journal of Engineering Innovation \&Research Volume 2, Issue 1, ISSN: $2277-5668$

[3]. C.S.Burrus, R.A.Gopinath, H.Guo, "Introduction to Wavelets and Wavelet Transforms", Prentice Hall, 1998, pp. 2-18.

[4]. $\quad$ D.L. Donoho, "De-noising by soft thresholding", IEEE Trans. on Info.Theory, pp. 933-936, 1993.

[5]. J. N. Ellinas, T. Mandadelis, A. Tzortzis, L. Aslanoglou, "Image de-noising using wavelets", T.E.I. of Piraeus, Department of Electronic Computer Systems.

[6]. G. Strang, T. Nguyen, "Wavelets and Filter Banks", Wellesley,1997.

[7]. S. Grace Chang, Bin Yu and M. Vattereli, "Adaptive Wavelet Thresholding for Image Denoising and Compression", IEEE Trans. Image Processing, vol. 9, pp. 1532-1546,Sept. 2000.

[8]. M. Vattereli and J. Kovacevic, "Wavelets andSubband Coding”. Englewood Cliffs, NJ, Prentice Hall, 1995.

[9]. Maarten Jansen, "Noise Reduction by WaveletThresholding", volume 161. Springer Verlag, United States of America, 1 edition, 2001.

[10]. Yungang Zhang, Bailing Zhang, Wenjin Lu, "Image Denoising and Enhancement Based on Adaptive Wavelet Thresholding and Mathematical Morphology"

[11]. S. Mallat, "A Theory for multiresolution signal decomposition: The wavelet representation", IEEE Trans. Pattern Anal. Machine Intell., Vol. 11, pp. 674-693, July 1989.

[12]. WalidDabour, "Improved Wavelet Based Thresholding for Contrast Enhancement of Digital Mammograms", 2008 International Conference on Computer Science and Software Engineering, pp. 948 951. Wuhan, China, 2008.

[13]. Fahimuddin.Shaik, Dr.M.N.Giri Prasad, Dr. JayabhaskarRao, A.SomaSekhar, B.Abdul Rahim, "Medical Image Analysis of Electron Micrographs in Diabetic Patients UsingContrast Enhancement", 2010 International Conference on Mechanical and Electrical Technology, 978-1-4244-8102-6/10 () 2010 IEEE

[14]. Ali Ziaei, HojatollahYeganeh, KarimFaez, SamanSargolzaei, "ANovel Approach For Contrast Enhancement In Biomedical ImagesBased On Histogram Equalization" 2008 International Conference OnBio Medical Engineering And Informatics”, Vol I, Pp. 855-858,_27-30, May 2008.

[15]. T. K. Kim, J. K. Paik, and B. S. Kang, "Contrast enhancement systemusing spatially adaptive histogram equalization with temporal filtering,", IEEE Trans. Consumer Electron., vol. 44, no. 1, pp. 82-87, Feb. 1998.

[16]. J. Tang, E. P. Peli, and S. Acton, "Image enhancement using a contrastmeasure in the compressed domain,” IEEE Signal Process. Lett., vol.10, no. 10, pp. 289-292, Oct. 2003.

[17]. I. Jafar and H. Ying, "Image contrast enhancement by constrainedvariational histogram equalization", in Proc. IEEE EIT, May 2007, pp.120-125.

[18]. Chuanwei Sun, Hong Liu, Jingao Liu, “An Image Enhancement Method for Noisy Image”, 978-14244-5858-5/10 @2010 IEEE, ICALIP2010. 


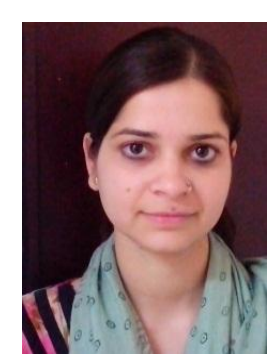

Saumya Dubey is presently pursuing her M. Tech. Studies in the Department of Electronics and Communication Engg., Amity University Uttar Pradesh, Lucknow, India. She received her M.Sc. Degree in Electronics from Kanpur University, Uttar Pradesh, India, in 2009. She has an online publication in reputed journal and actively involved in the field of Technical Education as a research scholar.

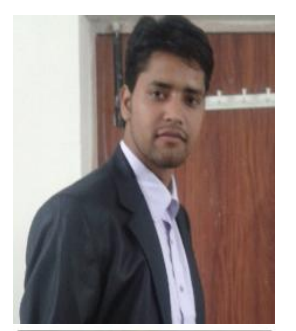

Deepak Tiwari is currently pursuing his M.Tech. Studies in the Department of Electronics and Communication Engineering., Amity University Uttar Pradesh,Lucknow,India. He received his B. Tech. Degree in Electronics and Communication from PunjabTechnical University, Jalandhar, India, in 2011. He has been actively involved in the field of Technical Education as a research scholar. He has an offline publication and three online publications in reputed journals.

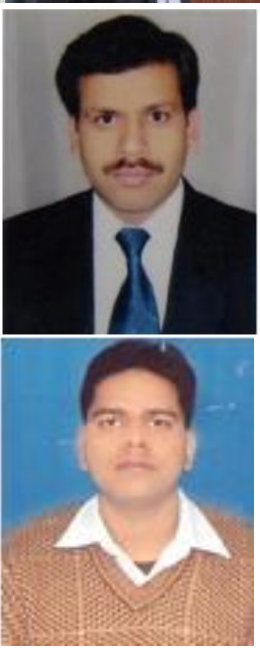

Dr. O. P. Singh obtained his Ph. D in Electronics Engineering for IT, BHU. He has over sixteen years experience and presently working as Professor and Head Department of Electronics \& Communication Engineering, Amity School of Engineering \& Technology, Amity University Uttar Pradesh Lucknow Campus. He is life member of Indian Society of Remote Sensing (ISRS) and Material Research Society of India (MRSI).He has presented several papers in National Seminar/Conferences and also published papers in National/international Journals. His area of interest includes Microwave Remote Sensing Digital System, Signal Processing.

Ashish Dixit is presently Asstt.Prof. in the department of Electronics and Communication Engg., Amity University Uttar Pradesh, Lucknow,India. He received his M.Tech. Degree in VLSI Design from CDAC (Center for Development of Advance Computing), Mohali, and he is Perusing Phd. from Uttrakhand Technical University, Dehradun. He is a member of Indian Microelectronics Society (Punjab University Chandigarh). He has been actively involved in the field of Technical Education as Academician, Researcher, Teacher, Planner and Administrator. He has more than seven years teaching experience. He was a Guest faculty for VLSI Design workshop conducted by Singhania University, Rajasthan on $14^{\text {th }}$ to $17^{\text {th }}$ March, 2008 and Paper setter of various Universities. He is an author of several books. He has guided several M.Tech. and B.Tech. students in Electronics Engg. discipline. He has also co-ordinated Front End VLSI design programme. 